\title{
Vers une politique européenne de l'enseignement des langues
}

Introduction

Towards a European policy for language teaching

\section{Roger Pilhion}

\section{OpenEdition}

\section{Journals}

Édition électronique

URL : http://journals.openedition.org/ries/325

DOI : $10.4000 /$ ries.325

ISSN : 2261-4265

\section{Éditeur}

Centre international d'études pédagogiques

Édition imprimée

Date de publication : 1 avril 2008

Pagination : 27-35

ISBN : 978-2-85420-572-5

ISSN : $1254-4590$

\section{Référence électronique}

Roger Pilhion, «Vers une politique européenne de l'enseignement des langues », Revue internationale d'éducation de Sèvres [En ligne], 47 | avril 2008, mis en ligne le 24 juin 2011, consulté le 30 avril 2019. URL : http://journals.openedition.org/ries/325 ; DOI : 10.4000/ries.325 


\title{
Vers une politique européenne de l'enseignement des langues
}

\author{
Roger Pilhion
}

Alors même que les États membres de l'Union européenne sont attachés à leurs prérogatives en matière éducative, force est de reconnaître que l'enseignement des langues étrangères dans les systèmes éducatifs s'inscrit de plus en plus dans une dynamique européenne.

\section{LE MULTILINGUISME, UNE RÉALITÉ EUROPÉENNE}

La connaissance de plusieurs langues, outre sa langue maternelle, devient aujourd'hui, de plus en plus, une exigence pour beaucoup d'Européens, en particulier pour les jeunes, dans la vie quotidienne comme dans la vie professionnelle: pour l'ouverture au monde, le dialogue interculturel, la tolérance, la mobilité au cours de la formation et sur le marché de l'emploi, pour construire la société de la connaissance, pour atteindre un bon niveau de compétitivité. Beaucoup d'Européens s'accordent aujourd'hui sur la nécessité pour chaque citoyen de maîtriser au moins une langue étrangère, voire deux, en plus de sa langue maternelle, et les systèmes éducatifs doivent contribuer à relever ce défi.

Il est vrai que le multilinguisme est un des fondements de l'Union européenne. De nombreuses langues cohabitent dans l'espace européen et la plupart des citoyens entretiennent avec leur langue, qu'elle soit nationale ou minoritaire au sein de leur pays, une relation identitaire. Ne dit-on pas que la langue est l'âme d'un peuple? L’Union européenne s'est bâtie sur le respect de cette diversité. Dès 1958, le Conseil des ministres de la Communauté stipulait que les langues officielles des pays signataires du traité de Rome seraient les langues officielles de la CEE. Récemment encore, deux États, pourtant très largement anglophones, ont réaffirmé leur attachement à ce principe: Malte, lors de son adhésion en 2004, en demandant la reconnaissance du maltais comme langue officielle, et l'Irlande, qui, après y avoir renoncé en 1973 lors de son adhésion, a demandé la reconnaissance officielle du gaélique, devenue effective en 2007. 


\section{DES INITIATIVES EUROPÉENNES EN FAVEUR DE L'ENSEIGNEMENT DES LANGUES}

L'enseignement des langues étrangères fait débat depuis longtemps en Europe.

Les travaux de la Division des politiques linguistiques du Conseil de l'Europe ont joué un rôle précurseur et se sont peu à peu imposés comme référence auprès des spécialistes de l'enseignement des langues dans les pays européens.

La publication du Niveau Seuil en 1975, d'abord dans sa version anglaise (Threshold level), puis en français l'année suivante, constitue une étape historique. Ce document établit un système européen d'unités capitalisables dans l'apprentissage des langues. Les niveaux seuil ont considérablement influencé de nombreux programmes nationaux et ont permis d'élaborer des manuels de langue se référant à une approche communicative, centrée sur l'élève.

Le Portfolio européen des langues a été élaboré et expérimenté par la Division des politiques linguistiques de 1998 à 2000. Il a été officiellement lancé pendant l'Année européenne des langues en 2001. Cet outil est un instrument d'auto-évaluation qui permet à chacun de noter ses connaissances linguistiques et interculturelles. L'objectif est de valoriser les compétences plurilingues tout en favorisant une réflexion sur son propre apprentissage.

La publication du Cadre européen commun de référence pour les langues (CECRL) en 2001 est l'aboutissement d'un processus de réflexion et de recherche engagé en 1991 et d'une large consultation. Ce document représente une base commune pour l'élaboration de programmes de langues étrangères, de manuels et d'examens. Ses six niveaux de compétence en langue deviennent des outils permettant une approche de l'évaluation commune à toutes les langues, dont les systèmes éducatifs s'emparent progressivement.

En 1994 enfin, le Conseil de l'Europe crée à Graz le Centre européen pour les langues vivantes, dans le cadre d'un accord partiel élargi aujourd'hui porté à trente-trois pays, pour la diffusion de ces politiques linguistiques, la constitution de réseaux de spécialistes, l'appui à l'innovation dans l'enseignement des langues et la formation des enseignants.

La Commission européenne s'est intéressée à ce dossier un peu plus tard, avec la création du programme Lingua, en 1989.

En 1995, des recommandations préconisent l'amélioration et le renforcement de l'apprentissage des langues. Ainsi, le conseil «Éducation» de l’Union européenne adopte le 31 mars 1995 une résolution concernant l'amélioration de la qualité et la diversification de l'apprentissage et de l'enseignement des langues au sein des systèmes éducatifs de l'Union européenne. 
Ce texte fait de «la promotion du pluralisme linguistique ... l'un des enjeux majeurs de l'éducation».

L’année 2001 est déclarée Année européenne des langues par le Conseil de l'Europe et l'Union européenne et rencontre un réel succès.

En 2002, la connaissance des langues fait partie des objectifs stratégiques du programme Education et formation 2010 de l'Union européenne. Un groupe permanent d'experts de langue où siègent des représentants des pays membres et des pays candidats est alors constitué. En mars de la même année, les chefs d'État et de gouvernement réunis à Barcelone demandent «qu'une action soit menée pour améliorer la maîtrise des compétences de base, notamment par l'enseignement d'au moins deux langues étrangères dès le plus jeune âge...». C'est ce même Sommet qui décide de la mise au point d'un indicateur des compétences linguistiques.

En 2004, le plan d'action de la Commission européenne 2004-2006 intitulé Promouvoir l'apprentissage des langues et la diversité linguistique voit le jour.

En 2005, pour la première fois, le multilinguisme fait officiellement partie des attributions d'un commissaire européen. Le Slovaque M. Jan Figel, est ainsi nommé commissaire européen «Éducation, Formation, Culture et Multilinguisme». La même année, la Commission présente une communication sur le «nouveau cadre stratégique pour le multilinguisme».

En 2006, un second groupe, constitué d'inspecteurs de langue des pays membres (ou de représentants exerçant des fonctions comparables), est créé par la Commission européenne.

En 2007, le portefeuille du multilinguisme devient le mandat unique du premier commissaire roumain, M. Leonard Orban. Le projet de création d'un indicateur européen des compétences linguistiques fait l'objet, la même année, d'un appel d'offres.

Au Programme d'éducation et de formation tout au long de la vie qui constitue le principal programme de financement européen dans le domaine de l'éducation pour la période 2007-2013 s'ajoute un programme transversal dont une des quatre activités représente la promotion de l'apprentissage des langues.

Fait remarquable: ces initiatives interviennent à la suite de décisions impulsées et validées par les États mais elles influencent, de fait et de plus en plus, les politiques nationales en matière éducative, faisant de l'enseignement des langues en Europe un cas particulier - et, qui sait, pionnier?

Les enseignants de langues et l'encadrement à un niveau intermédiaire n'ont pourtant pas tous nécessairement conscience de la dimension européenne des évolutions qu'ils sont appelés à constater et à mettre en œuvre sur le terrain. 


\section{L'ENSEIGNEMENT DES LANGUES EN EUROPE: QUELQUES REPÈRES ${ }^{1}$}

Quelques éléments saillants, en matière d'enseignement des langues en Europe, méritent d'être relevés, en préambule de ce dossier.

Les États proposent quasiment tous un enseignement obligatoire d'une langue vivante étrangère, à l'exception de l'Irlande où l'enseignement d'une langue est pourtant généralisé et la plupart, avec quelques exceptions dont le Royaume-Uni, proposent deux langues obligatoires, voire l'enseignement d'une troisième langue optionnelle. Toutefois, ces deux langues vivantes ne sont pas toujours prises en compte dans le diplôme de fin d'études secondaires. C'est généralement le cas pour la première langue (LV1); cela reste assez rare pour la seconde (LV2). Et dans de nombreux pays, les épreuves restent assez traditionnelles, les compétences orales n'étant pas nécessairement évaluées.

Les langues les plus enseignées sont l'anglais, le français, l'allemand, l'espagnol, l'italien et le russe, cette dernière langue étant surtout présente dans les pays d'Europe centrale.

L'anglais langue étrangère occupe aujourd'hui une position dominante de fait dans la plupart des systèmes éducatifs des pays non anglophones. Certains États, la Suède par exemple, tendent même à considérer son enseignement comme faisant partie intégrante des savoirs fondamentaux, ce qui, de facto, revient à ne plus considérer l'anglais comme une langue étrangère au même titre que les autres.

La place de deuxième langue est partagée, selon les zones géographiques et les traditions pédagogiques, entre le français, l'allemand et, dans une moindre mesure, l'espagnol et le russe.

L'effort de diversification de l'offre est inégal selon les systèmes éducatifs, celle-ci pouvant aller jusqu'à une vingtaine de langues, mais la demande sociale favorise partout un nombre limité de langues. On constate, par ailleurs, dans plusieurs pays, un intérêt nouveau et marqué pour le chinois.

La durée de l'apprentissage obligatoire d'une ou de deux langues varie fortement, de trois ans (Pays de Galles par exemple) à treize ans (Luxembourg) pour la LV1. Pour la LV2, celle-ci varie de deux à trois ans en Pologne, de deux à quatre ans en Allemagne, jusqu'à six ou sept ans (Finlande, France, Suède par exemple) et même jusqu'à douze ans au Luxembourg. On observe, dans certains pays, la volonté de réduire l'écart entre l'enseignement de la première et de la deuxième langue vivante étrangère enseignée, en particulier dans le cadre d'options (c'est, par exemple, le cas en Allemagne et en France), mais cet objectif reste difficile à atteindre.

1. On pourra se reporter pour des analyses plus précises aux enquêtes Eurydice figurant dans les «Chiffres clés de l'enseignement des langues à l'école en Europe.»" 
Beaucoup d'États ont avancé le début de l'apprentissage d'une langue étrangère au primaire et proposent celui-ci dès l'âge de six ans (Luxembourg, Portugal) ou de sept à huit ans (Allemagne, Bulgarie France, Finlande, Suède, par exemple). Le Royaume-Uni, qui propose encore un enseignement des langues à partir de dix à onze ans (dix en Ecosse, onze en Angleterre), s'apprête à le faire. D'autres pays n'ont pas encore pris ces dispositions, la Pologne notamment qui propose un enseignement d'une langue vivante étrangère à partir de dix ans.

L'enseignement disciplinaire en langue étrangère, fondé sur l'enseignement de matières par l'intégration d'une langue étrangère (EMILE), le plus généralement dans le second cycle, a connu un réel développement dans beaucoup de pays, surtout à partir des années quatre-vingt-dix. Il constitue aujourd'hui, avec l'enseignement précoce, une des voies privilégiées pour améliorer les compétences des élèves.

On observe, depuis le début des années 2000, une tendance de certains systèmes éducatifs à recourir à des certifications étrangères, reconnues internationalement et harmonisées, pour certaines d'entre elles, sur le CECRL. Mais celles-ci ne remplacent pas les examens nationaux de langues.

La mobilité joue un rôle de plus en plus important dans l'amélioration des compétences des enseignants de langues. Des programmes européens et nationaux ont été créés à cet effet.

Les échanges bilatéraux d'assistants de langue, quoique très anciens, et le programme européen d'assistants apportent une contribution significative à la qualité de cet enseignement, en mettant des élèves en contact avec des locuteurs natifs. Plus de vingt mille jeunes participent chaque année à ces programmes.

\section{Orientations politiques, RÉFLEXIONS PÉDAGOGIQUES ET BONNES PRATIQUES}

Ce numéro intitulé «Enseigner les langues: un défi pour l'Europe » est le sixième à être consacré à la question des langues par la Revue internationale d'éducation de Sèvres ${ }^{2}$. Il se propose d'illustrer ces évolutions en présentant, d'une part, des analyses politiques et des réflexions pédagogiques prospectives s'inscrivant dans un cadre supranational et, d'autre part, des initiatives significatives et des pratiques innovantes, prises au niveau des politiques nationales.

2. La Revue internationale d'éducation de Sèvres a publié sur cette thématique: $n^{\circ} 3$ (1994): «les langues régionales et l'Europe »; $n^{\circ} 7$ (1995): «enseignements bilingues »; $n^{\circ} 9$ (1996): «des langues vivantes à l'école »; $n^{\circ} 19$ (1998): «langue maternelle, langue d'enseignement»; $n^{\circ} 33$ (2003): «l'enseignement des langues vivantes à l'étranger, enjeux et stratégies ». 
Pour ce faire, il a été fait appel au commissaire Leonard Orban qui souligne l'importance politique accordée par la Commission européenne au multilinguisme dans un article qui ouvre ce dossier. La politique du multilinguisme vise à ouvrir l'Europe sur le monde et les Européens sur eux-mêmes. La protection du patrimoine linguistique européen et l'égale valeur et dignité des langues, y compris des langues d'origine des migrants, constituent à ses yeux un enjeu essentiel. C'est pourquoi, l'Europe a besoin d'enseignants compétents, de programmes appropriés dans le domaine des langues et d'une approche ambitieuse de l'enseignement des langues, autant de thématiques qui sont développées dans cet article qui se termine par l'annonce d'une communication en septembre 2008 sur le multilinguisme et sa contribution au développement économique, social et culturel de l'Union.

La formation des enseignants de langues est, bien sûr, au cœur de ces réflexions car elle conditionne largement la qualité de l'enseignement et donc l'amélioration des compétences linguistiques des Européens. Bien que cette question relève de la compétence exclusive des États, la Commission a financé une étude intitulée Profil européen de la formation des enseignants de langues, publiée en 2004, dont le professeur Michael Kelly, qui en a assuré la coordination, présente les enjeux et les difficultés de réalisation. Un résumé du Profil est, en outre, proposé en annexe de son article. Partant du constat d'une grande diversité des situations et des contenus de formation, l'étude s'est attachée à définir des concepts communs et un cadre de référence visant à favoriser les échanges d'idées et les convergences volontaires. À ce stade, ses retombées ne sont pas connues mais il ne fait pas de doute que les responsables nationaux de la formation des enseignants de langue peuvent tirer le plus grand bénéfice de cette véritable boîte à idées, née de la confrontation et de la comparaison des expériences conduites dans une trentaine de pays ${ }^{3}$.

Deux articles rendent ensuite compte de l'introduction du CECRL dans divers systèmes éducatifs européens.

Le premier, rédigé par Francis Goullier, expert auprès du Conseil de l'Europe et représentant de la France au sein du groupe d'experts de langue à la Commission européenne, tire les premiers enseignements d'une démarche qui touche un nombre important d'États. Rappelant que l'utilisation du CECRL doit être contextualisée pour avoir toute sa pertinence, l'auteur analyse l'utilisation très diversifiée qui en a été faite, pour définir des objectifs d'apprentissage par référence à l'échelle de niveaux ainsi que pour évaluer le niveau de compétences des élèves. Il souligne également l'impact de cet outil sur les programmes d'enseignement des langues et, dans une moindre mesure, sur les standards de formation, mais regrette les faibles avancées dans la promotion des

3. Une proposition vient, au demeurant, d'être soumise à la Commission, à l'initiative du Centre international d'études pédagogiques, proposant l'organisation d'une université d'été en 2009 qui pourrait réunir sur ce thème des responsables de la formation des enseignants de langues des pays concernés par cette étude. 
valeurs portées par le Conseil de l'Europe à travers le CECRL, telles que la promotion de la diversité et le développement de l'autonomie des apprenants, qui trouvent leur traduction, notamment, dans la notion de plurilinguisme.

L'article du professeur Cristina Avelino rend compte spécifiquement du cas du Portugal qui fut un des premiers pays à avoir introduit le CECRL dans son système éducatif, dès 2001. Le bilan contrasté de cette expérience est intéressant, dans la mesure où est démontrée a posteriori la nécessité, pour un pays donné, d'avoir une approche globale et commune à toutes les langues enseignées, pour la mise en œuvre d'une réforme de l'enseignement des langues intégrant cet outil.

La question de l'enseignement précoce des langues est abordée à travers l'exemple de la Finlande. Ursula Viita-Leskelä, formatrice à l'École normale Viikin d'Helsinki, présente une expérience très aboutie dans un pays dont les bons résultats scolaires sont connus et où cet enseignement semble aller de soi et ne pas faire débat comme dans d'autres pays. Proposée généralement dès la troisième année de l'école fondamentale, la première langue vivante étrangère, l'anglais pour la très grande majorité des élèves (car contrairement à la Suède voisine, la Finlande privilégie la diversification de l'offre), est enseignée avec une approche adaptée aux enfants qui favorise la motivation, par des professeurs bien formés et selon une progression qui permet de prendre en compte les premiers acquis dans l'ensemble du cursus. Les acquis à la fin du second cycle semblent assez remarquables puisque $60 \%$ des élèves atteignent le niveau B2 et $25 \%$ le niveau C1. Un encadré de Catherine Clément, responsable du Département des langues étrangères du CIEP, qui signe par ailleurs l'article consacré à la France, brosse un panorama de l'enseignement précoce des langues en Europe.

La question de l'enseignement bilingue est illustrée par la Bulgarie. Alors que l'enseignement disciplinaire en langue étrangère est un phénomène relativement récent en Europe occidentale, on ne peut qu'être frappé par l'expérience qui remonte aux années 1950 relatée par Veselina Ganeva, expert d'État au ministère de l'Éducation et des sciences, elle-même ancienne élève d'un de ces «lycées de langues", comme on les appelait alors. Parmi les initiatives les plus originales décrites dans cet article, on retiendra particulièrement la classe intensive de langue qui propose, sur une année scolaire, une formation linguistique intensive pouvant aller jusqu'à vingt-deux heures de cours par semaine dans les écoles bilingues. Les élèves sont ainsi préparés à suivre des cours dans différentes disciplines telles que l'histoire, la biologie, la géographie, la chimie, la physique, la philosophie, les nouvelles technologies et les mathématiques, dispensés en langue étrangère dans les quatre dernières années du cycle secondaire. L'ambition affichée dans le Programme national de développement de l'enseignement secondaire pour les années 2006 à 2015 de généraliser la classe intensive de langue à tous les établissements scolaires mérite également d'être relevée. 
L'introduction de certifications étrangères est présentée dans un article qui rend compte de l'expérience conduite avec le DELF scolaire, diplôme national de français langue étrangère harmonisé sur le CECRL dans le Palatinat du Nord-Westphalie, Land le plus peuplé d'Allemagne (dix-sept millions d'habitants; trois millions d'élèves). Cet article présente un exemple particulièrement structurant de coopération entre la France et cet État allemand dans le domaine de l'enseignement du français. À l'heure où la question de l'évaluation est au cœur des préoccupations européennes, les autorités ministérielles du Land ont recours au DELF scolaire sur la base du volontariat des familles. Cette mesure n'est pas sans effet sur les pratiques d'enseignement, la motivation des élèves, la formation des enseignants même et donc la qualité de l'enseignement du français, ainsi qu'en témoigne le succès croissant de cette expérience. Un encadré élaboré par Christophe Fauchon, attaché de coopération pour le français à l'ambassade de France à Berlin, élargit la perspective à toute l'Allemagne.

S'agissant de la France, dont l'enseignement des langues a connu de fortes évolutions depuis 2000 et où un plan de rénovation de l'enseignement des langues mettant l'accent sur "l'amélioration des capacités de communication, notamment à l'oral» et «la préparation à la mobilité européenne et internationale» est mis en ouvre depuis 2005, il a paru intéressant de présenter les mesures d'accompagnement en ligne, décidées par le ministère de l'Éducation nationale, en appui à deux des principales innovations, l'introduction d'un enseignement obligatoire d'une langue vivante à l'école primaire et le développement de l'enseignement disciplinaire en langues étrangères dans les lycées comportant des «sections européennes ou de langues orientales» qui ont abouti à la création de deux sites internet Primlangues et Emilangues offrant des ressources pour l'enseignement de et en sept langues.

Ce dossier se termine par la présentation par Anders Hingel, chef de l'Unité «Analyses et prospective» à la direction générale de l'éducation et de la culture de la Commission européenne, et Marguerite Kuzma, du projet de mise en place d'un Indicateur européen des compétences linguistiques, dont la décision formelle remonte au Conseil européen de Barcelone de 2002 et qui, au terme d'une procédure d'appels d'offres, vient d'être mis en chantier en janvier 2008. Cette première enquête européenne sur les compétences linguistiques des jeunes Européens portera, dans un premier temps, sur les deux langues étrangères les plus enseignées dans chacun des pays, parmi les cinq langues officielles des États membres de l'Union européenne les plus enseignées en Europe, l'anglais, le français, l'allemand, l'espagnol et l'italien. Chaque État devra donc déterminer les deux langues qu'il décidera d'évaluer parmi ces cinq et pourra, le cas échéant, étendre son enquête aux autres langues. L'enquête portera sur trois compétences linguistiques, l'expression écrite et la compréhension écrite et orale d'élèves inscrits en dernière année du premier cycle de l'enseignement 
secondaire ou en deuxième année du deuxième cycle de l'enseignement secondaire, si une deuxième langue étrangère n'est pas étudiée dans le premier cycle. Un outil d'évaluation mesurant une progression continue des compétences, exprimées sur les quatre premiers niveaux du CECRL (niveaux $\mathrm{A} 1$ à $\mathrm{B} 2$ ) sera élaboré pour ces cinq langues par le consortium ayant remporté l'appel d'offres. On pourra, bien sûr, regretter que, pour des raisons d'organisation, l'expression orale en ait été écartée, dans un premier temps. Il n'en reste pas moins que ce futur PISA des langues qui devrait être mis en place en 2010 est particulièrement attendu.

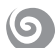

Une question reste ouverte: ces évolutions permettront-elles de relever le défi de la sauvegarde des identités qui s'expriment à travers ces multiples langues, et auquel l'Europe est confrontée? Ou finira-t-elle, au nom d'un pragmatisme simplificateur, à s'accommoder du tout anglais comme lingua franca? La Commission européenne contient en germe cette contradiction, puisqu'elle promeut le multilinguisme, tout en ayant recours, de plus en plus fréquemment, à une seule langue de communication dans les réunions où l'interprétariat ne peut être assuré et, très souvent, dans les relations intra-européennes et internationales. 
